\section{ACTM-838: A MICROBIAL-BASED IMMUNOTHERAPY THAT DELIVERS COMBINATION IL-15 + ENGINEERED STING TO TUMOR-RESIDENT APCS AFTER IV DOSING IN T-CELL EXCLUDED SOLID TUMORS}

${ }^{1}$ Haixing Kehoe*, 'Alexandre lannello, ${ }^{1}$ Keith Cheung, ${ }^{1}$ Bret Peterson, ${ }^{2}$ Marie Marotel, ${ }^{1}$ Sara Tribble, ${ }^{1}$ Hailey He, ${ }^{2}$ Jonathan Hodgins, ${ }^{2}$ Michele Ardolino, ${ }^{1}$ Christopher Rae, ${ }^{1}$ Christopher Thanos, 'Laura Glickman. ${ }^{1}$ Actym Therapeutics, Inc, Berkeley, CA, USA;

2University of Ottawa, OHRI, Ottawa, Canada

Background In a metastatic setting, systemically-administered therapies that overcome the immunosuppressive tumor microenvironment to promote T-cell recruitment and T-cell cytolytic function will be required to elicit durable anti-tumor immunity. To accomplish this, the STACT (S. Typhimurium-Attenuated Cancer Therapy) platform was developed. STACT is a live bacterial product that has been highly modified using precision genome editing for the following properties: (1) enhanced tolerability after IV dosing, (2) tumor-specific enrichment, (3) phagocytosis by tumor-resident antigen-presenting cells (APCs) with a lack of epithelial cell infectivity, (4) multiplexed genetic cargo delivery, and (5) attenuation of bacterial pathways that impair $\mathrm{CD} 8+\mathrm{T}$-cell function. An extensive screening campaign was performed to identify ideal encoded immunomodulatory payload combinations delivered by STACT for efficacy against T-cell excluded tumors.

Methods Chromosomal edits to the STACT platform strain were made using PCR. A panel of immunomodulatory proteins, including cytokines, type I interferon (IFN)-inducing factors, co-stimulatory receptors, checkpoint antibodies and TGF $\beta R$-Fc decoys were tested for combinatorial potency using STACT. An engineered STING (eSTING) was designed through an extensive protein engineering campaign to identify optimal variants. Combinations were evaluated in primary human APCs using in vitro functional assays, where STACT IL-15R $\alpha$-IL-15 (IL-15) + eSTING (ACTM-838) emerged as a lead candidate. ACTM-838 was then evaluated in multiple murine tumor models for therapeutic efficacy and mechanism, as well as tolerability in rodents and primates after systemic administration.

Results Combinatorial target profiling led to the discovery of ACTM-838, a STACT encoding IL-15 + eSTING. In vitro, ACTM-838 payloads synergistically produced high levels of type I IFN and T-cell recruitment and activation factors from primary human APCs. In vivo, ACTM-838 demonstrated a high degree of complete tumor responses that were entirely CD8 + T-cell dependent. In an autochthonous breast cancer model that lacks any significant lymphocyte infiltrate, ACTM838 was able to uniformly enrich in each spontaneous lesion to high levels after IV dosing and resulted in significant CD8 + T-cell infiltration. In primates, ACTM-838 was well-tolerated, rapidly cleared, and elicited minimal cytokine response after IV dosing.

Conclusions ACTM-838 is a highly attenuated, precision genome-engineered bacterial immunotherapy that delivers IL$15+$ eSTING to phagocytic APCs of the solid tumor microenvironment after systemic administration. In preclinical studies, ACTM-838 promotes CD8 + T-cell mediated tumor clearance in T-cell excluded tumors and elicits durable antitumor immunity, and is well tolerated in primates. Based on these data, ACTM-838 was nominated for clinical development and has entered cGMP manufacturing and IND-enabling studies.

Ethics Approval All animals were used according to protocols approved by an Institutional Animal Care and Use Committee and maintained in specific pathogen-free conditions in a barrier facility.

http://dx.doi.org/10.1136/jitc-2021-SITC2021.853 